\title{
Avaliação in vitro da força liberada por elásticos em cadeia
}

Andréa Fonseca Jardim da Motta*, Adriana de Alcantara Cury-Saramago*, Lincoln Issamu Nojima**

\section{Resumo}

Objetivo: avaliar in vitro e comparar a redução de intensidade das forças liberadas por três grupos de elásticos em cadeia, com diferentes configurações, de um mesmo fabricante, medidas em intervalos de tempo preestabelecidos. Métodos: os segmentos de elásticos em cadeia foram alongados e mantidos ativados durante o experimento com o auxílio de um dispositivo desenvolvido especialmente para esse fim e para possibilitar a leitura das forças. A avaliação da degradação da força foi realizada medindo-se a intensidade da força ao longo do tempo, e calculando-se o percentual de força perdida em relação à força inicial, em cada intervalo de tempo e para cada amostra testada. Resultados e Conclusões: os dados coletados foram submetidos a análises estatísticas e os resultados demonstraram que, nos momentos seguintes ao inicial, a intensidade da força variou, dentro dos grupos e entre os grupos, nos diferentes momentos. A leitura dos valores das forças remanescentes em cada momento, comparada à referência da força inicial, resultou em valores diferentes, com significância estatística, em todas as comparações efetuadas dentro de cada grupo de elásticos (curto, médio, longo). Após a análise dos resultados das comparações entre os grupos de elásticos em cadeia, conclui-se que, apesar de verificados alguns resultados estatisticamente significativos para a comparação das magnitudes das forças liberadas em cada momento, essas diferenças parecem não ter expressão clínica, demonstrando que, aparentemente, o espaçamento entre os elos não representa uma característica clinicamente significativa na degradação da força ao longo do tempo.

Palavras-chave: Aparelhos ortodônticos. Materiais dentários. Elastômeros.

\section{INTRODUÇÃO}

A Ortodontia baseia-se no princípio de que uma força externa aplicada intencionalmente sobre um dente é capaz de promover o movimento dentário como resultado da reação biológica dos tecidos dentoalveolares. A força ortodôntica ideal requerida para alcançar o movimento dentário é alvo de alguma contro-

Como citar este artigo: Motta AFJ, Cury-Saramago AA, Nojima LI. Avaliação in vitro da força liberada por elásticos em cadeia. Dental Press J Orthod. 2011 Nov-Dec;16(6):36.e1-8. vérsia; mas, comumente, aceita-se que forças leves e contínuas sejam consideradas ótimas ${ }^{4}$. O conhecimento da biomecânica ortodôntica permite a utilização de uma variedade de sistemas de força capaz de promover a força ótima, com o máximo de velocidade de movimento, sem desconforto para o paciente ou danos aos tecidos de suporte.

"Os autores declaram não ter interesses associativos, comerciais, de propriedade ou financeiros que representem conflito de interesse, nos produtos e companhias descritos nesse artigo.

* Doutora em Ortodontia pela UFRJ. Professora Adjunta da Universidade Federal Fluminense.

** Professor Adjunto de Ortodontia da Universidade Federal do Rio de Janeiro. 
A utilização dos elásticos em cadeia na Ortodontia data dos idos de $1960^{3,8}$ e cresce ao longo do tempo, devido às muitas vantagens que apresenta, dentre as quais as possibilidades de substituir ligaduras metálicas, arcos de fechamento, molas abertas ou fechadas; permitindo o fechamento de espaços, a correção de rotações e movimento de caninos em direção distal ${ }^{19}$. Além disso, os elásticos em cadeia são econômicos, confortáveis, facilmente aplicados e removidos, o que reduz o tempo de trabalho além de não requerer a cooperação do paciente ${ }^{23}$. São também relativamente higiênicos e, na maioria das vezes, compativeis com a mucosa bucal; fatores que contribuem para o seu alto grau de aceitação junto aos ortodontistas ${ }^{4,7,12,24}$. No entanto, apresentam a desvantagem da incapacidade de desenvolver, ao longo do tempo, intensidade de força contínua suficiente para movimentar dentes ${ }^{3}$.

As propriedades mecânicas dos elásticos em cadeia modificam-se com o tempo, pois sofrem alteração em função da quantidade de extensão a que são submetidos, absorvem água e saliva, sofrem deformação permanente e, ainda, são sensíveis ao $\mathrm{pH}$ salivar e à variação de temperatura no meio bucal ${ }^{8}$. $\mathrm{O}$ tamanho das cadeias elásticas - descrito como curto, médio ou longo - , assim como a sua configuração, também parecem influenciar em seu comportamento $8,9,10,28$. Segundo alguns autores, os elásticos com configuração curta, sem espaço entre os elos, mantém maior percentual de força ao longo do tempo ${ }^{3,8}$.

Os elásticos em cadeia, geralmente, são instalados e substituídos em intervalos de três a quatro semanas ${ }^{6}$. Após esse período, parecem permanentemente alongados e descoloridos ${ }^{1}$. Também não devem ser empregados continuamente por mais de seis semanas, tanto pela incapacidade de manutenção das propriedades mecânicas quanto pela prevenção de acúmulo de placa bacteriana sobre o material ${ }^{12}$.

Segundo alguns autores, a maior diminuição da força liberada pelos elásticos em cadeia ocorre na primeira hora ${ }^{15,16,18}$, mas são relatadas perdas de $33 \% 10,15,19$ até $50 \%{ }^{11}$ dessa força nas primeiras 24 horas após sua aplicação. Outros autores verificaram perdas de até
$73 \%$ nesse mesmo período, seguido de três semanas liberando forças razoavelmente constantes ${ }^{1,27}$.

Para a realização do tratamento ortodôntico de forma satisfatória, é necessário conhecer os atributos e propriedades dos elásticos ortodônticos, assim como a monitoração da quantidade de força liberada em diferentes intervalos de tempo. Muitas pesquisas foram realizadas a respeito das propriedades mecânicas dos elásticos em cadeia, mas a literatura ainda é contraditória, principalmente com relação ao grau de diminuição da intensidade da força com o tempo ${ }^{4,10,13-18}$.

Através da presente pesquisa objetivou-se avaliar in vitro e comparar a diminuição da força inicialmente liberada por três grupos de elásticos em cadeia, com diferentes espaçamentos entre os elos (curto, médio e longo), de um mesmo fabricante, medidos em intervalos de tempo preestabelecidos.

\section{MATERIAL E MÉTODOS}

Para a realização dessa pesquisa, foram selecionados elásticos em cadeia da cor cinza, fabricados pela Dental Morelli (Sorocaba/SP, Brasil), com três configurações, relacionadas aos tamanhos diferentes do espaçamento entre os elos: curto (60.05.501), médio (60.05.511) e longo (60.05.521). Antes de serem testados, os elásticos foram mantidos em suas embalagens originais, segundo a recomendação do fabricante.

A amostra foi composta por três grupos de 20 segmentos de elásticos cada, de acordo com a configuração: grupo Curto; grupo Médio e grupo Longo. As cadeias elásticas testadas foram cuidadosamente removidas dos carretéis, sem serem alongadas. Para todos os segmentos de elásticos, somente os elos centrais foram ativados, deixando-se um elo extra em cada extremidade, de modo a eliminar a possibilidade de dano acidental à cadeia durante o corte e remoção do carretel, fato que poderia tornar a estrutura frágil e predisposta à fratura, sem que tal dano fosse identificado visualmente.

Um dispositivo com uma base fixa e outra móvel, com ganchos nas extremidades, foi confeccionado especialmente para manter os elásticos 
ativados durante o experimento e permitir a leitura da magnitude das forças liberadas. As bases do dispositivo foram ajustadas para uma distância, entre os ganchos, de $20 \mathrm{~mm}$ de comprimento, com auxílio de um paquímetro digital (Starret Indústria e Comércio Ltda., Itu, São Paulo, número de série 001296), simulando a distância entre a asa mesial do braquete do canino e a asa distal do braquete do segundo pré-molar ${ }^{8,17}$. Em seguida, os segmentos de elástico em cadeia foram colocados no dispositivo e alongados buscando uma liberação de força inicial com magnitude aproximada de 200gf.

Foram realizadas medições nos seguintes intervalos de tempo: inicial; uma hora; vinte e quatro horas; uma, duas, três e quatro semanas após a única ativação dos elásticos.

A avaliação da degradação de força foi realizada medindo-se a intensidade de força ao longo do tempo e calculando-se o percentual de força perdida em relação à força inicial, em cada intervalo de tempo e para cada amostra testada.

Os dispositivos contendo os segmentos de elásticos ativados foram mantidos imersos em saliva artificial, dentro de estufa com temperatura controlada a $37 \pm 1^{\circ} \mathrm{C}$. Nos momentos imediatamente anteriores às medições, os dispositivos foram removidos do recipiente e, ao final, recolocados.

O nível da saliva artificial existente no recipiente foi verificado diariamente, para que os elásticos estivessem sempre cobertos por essa solução. A saliva artificial utilizada foi elaborada na farmácia da Universidade Federal do Rio de Janeiro.

A distância entre os ganchos da base fixa e da base móvel do dispositivo foi diminuída $0,5 \mathrm{~mm}$ por semana, de modo a simular o movimento dentário ortodôntico de um canino em direção distal ${ }^{5,11}$. Dessa forma, ao final da primeira semana a distância entre as bases foi diminuída para $19,5 \mathrm{~mm}$ e assim sucessivamente, até se chegar ao final do experimento, com uma distância de $18 \mathrm{~mm}$ entre as bases fixa e móvel do dispositivo.

\section{Tratamento estatístico}

O tratamento estatístico foi realizado comparando-se os valores de força liberada pelos elásticos, para verificar a existência de diferenças estatisticamente significativas entre os grupos (para cada um dos intervalos de tempo, separadamente) e dentro de cada grupo (considerando-se as diferenças entre as forças de um momento para o outro).

Para verificar a condição de normalidade dos dados, foram realizados testes de Shapiro-Wilk. A fim de verificar possíveis diferenças entre os três grupos, utilizou-se o teste paramétrico de Análise de Variância (ANOVA). Foi aplicado o teste de Levene quando os dados foram considerados normais, e o teste não paramétrico de Kruskal-Wallis nos grupos que não satisfizeram o critério de normalidade, seguido do teste de Mann-Whitney, para identificação das diferenças. Adotou-se o nível de 5\% de significância em todos esses testes descritos.

Testes não paramétricos de Friedman e Wilcoxon foram efetuados objetivando-se verificar as possíveis diferenças dentro de cada grupo, adotando-se o nível de $1 \%$ de significância.

\section{RESULTADOS}

$\mathrm{Na}$ Tabela 1 constam as médias e desvios-padrão da força liberada pelos elásticos dos grupos Curto, Médio e Longo, assim como o percentual médio de degradação da força, nos diferentes momentos.

$\mathrm{Na}$ Tabela 2 estão apresentados os níveis de significância para as diferenças médias, entre os grupos, quanto às intensidades de força liberada (gf), nos diferentes momentos.

Os valores médios da magnitude da força inicialmente liberada verificados para os grupos Curto, Médio e Longo foram 237,3gf, 227,3gf e 230,3gf, respectivamente. Constatou-se diferença estatisticamente significativa $(p<0,05)$ entre os valores da força liberada pelos elásticos curtos e pelos outros dois grupos de elásticos (médio e longo), e não houve diferença estatisticamente significativa $(p>0,05)$ entre os valores das forças desses últimos dois grupos de elásticos. 
TABELA 1 - Médias e desvios-padrão das forças liberadas pelos elásticos dos grupos Curto, Médio e Longo (em gf, ou gramas-força), e percentual médio de degradação da força, nos diferentes momentos.

\begin{tabular}{|c|c|c|c|c|c|}
\hline \multirow[b]{2}{*}{ Momento } & \multirow[b]{2}{*}{ Grupo } & \multicolumn{2}{|c|}{ Força liberada } & \multicolumn{2}{|c|}{ Degradação da força } \\
\hline & & $\begin{array}{c}\text { Média } \\
\text { (gf) }\end{array}$ & d.p. & $\begin{array}{c}\text { Média } \\
(\%)\end{array}$ & d.p. \\
\hline \multirow{3}{*}{ Inicial } & Curto & 237,25 & 11,64 & - & - \\
\hline & Médio & 227,25 & 11,29 & - & - \\
\hline & Longo & 230,25 & 7,52 & - & - \\
\hline \multirow{3}{*}{1 hora } & Curto & 230,00 & 12,25 & 3,06 & 1,73 \\
\hline & Médio & 218,25 & 10,04 & 3,92 & 2,13 \\
\hline & Longo & 219,25 & 7,99 & 4,78 & 1,66 \\
\hline \multirow{3}{*}{24 horas } & Curto & 193,25 & 11,50 & 18,55 & 2,48 \\
\hline & Médio & 182,25 & 6,97 & 19,69 & 3,44 \\
\hline & Longo & 185,75 & 6,34 & 19,32 & 1,61 \\
\hline \multirow{3}{*}{1 semana } & Curto & 148,75 & 10,24 & 37,29 & 3,23 \\
\hline & Médio & 148,25 & 7,66 & 34,68 & 3,65 \\
\hline & Longo & 149,50 & 8,41 & 35,08 & 2,75 \\
\hline \multirow{3}{*}{$\begin{array}{c}2 \\
\text { semanas }\end{array}$} & Curto & 136,25 & 11,11 & 42,61 & 2,86 \\
\hline & Médio & 130,75 & 7,66 & 42,44 & 2,46 \\
\hline & Longo & 134,00 & 6,81 & 41,79 & 2,54 \\
\hline \multirow{3}{*}{$\begin{array}{c}3 \\
\text { semanas }\end{array}$} & Curto & 112,25 & 10,82 & 52,74 & 3,08 \\
\hline & Médio & 116,25 & 7,59 & 48,85 & 2,10 \\
\hline & Longo & 120,00 & 6,69 & 47,89 & 2,28 \\
\hline \multirow{3}{*}{$\begin{array}{c}4 \\
\text { semanas }\end{array}$} & Curto & 102,75 & 8,19 & 56,72 & 2,21 \\
\hline & Médio & 96,25 & 6,86 & 57,63 & 2,41 \\
\hline & Longo & 109,75 & 5,95 & 52,35 & 1,71 \\
\hline
\end{tabular}

TABELA 2 - Níveis de significância para as diferenças médias das intensidades de força liberada (gf) entre os grupos, nos diferentes momentos.

\begin{tabular}{cccc} 
Momento & \multicolumn{3}{c}{ Grupos } \\
& Curto X Médio & Curto X Longo & Médio X Longo \\
\hline Inicial & $10^{*}$ & $7^{*}$ & $-3^{\text {ns }}$ \\
\hline 1 hora & $11,7^{*}$ & $10,75^{*}$ & $-1^{\text {ns }}$ \\
\hline 24 horas & $11^{*}$ & $7,5^{*}$ & $-3,5^{\text {ns }}$ \\
1 semana & $0,5^{\text {ns }}$ & $-0,75^{\text {ns }}$ & $-1,25^{\text {ns }}$ \\
\hline 2 semanas & $5,5^{\text {ns }}$ & $2,25^{\text {ns }}$ & $-3,25^{\text {ns }}$ \\
\hline 3 semanas & $-4^{*}$ & $-7,75^{*}$ & $-3,75^{*}$ \\
\hline 4 semanas & $-7^{*}$ & $6^{*}$ & $-13,5^{*}$
\end{tabular}

* = significativo ao nível de $5 \%$ de probabilidade, ns = não significativo.

Para a comparação, dentro de cada grupo de elásticos (Curto, Médio e Longo), dos valores das forças remanescentes nos diferentes momentos em relação ao inicial, aplicou-se os testes não paramétricos de Friedman e de Wilcoxon e os resultados apresentaram diferença estatisticamente significativa $(p<0,01)$ entre todas as comparações efetuadas.

A comparação dos grupos de elásticos, nos três primeiros momentos medidos (inicial, 1 hora, 24 horas), foi efetuada através da análise da variância (ANOVA), em virtude da satisfação do critério de normalidade pelos dados nesses momentos, seguindo-se a aplicação de métodos de comparações múltiplas para identificação das diferenças. Nos momentos seguintes ( 1 semana, 2 semanas, 3 semanas, 4 semanas), dada a não evidência de satisfação do critério de normalidade, a comparação dos grupos de elásticos foi procedida por métodos não paramétricos, especificamente a análise da variância de Kruskal-Wallis, seguida do teste de Mann-Whitney para identificação das diferenças.

Após transcorrida a primeira hora desde a ativação, os grupos Curto, Médio e Longo apresentaram, respectivamente, valores de 230gf, 218,25gf e 219,25gf. Conclui-se que, com significância estatística $(\mathrm{p}<0,05)$, após 1 hora a força liberada pelos elásticos curtos é maior do que a liberada por cada um dos outros dois grupos de elásticos (médios e longos) e que não há diferença estatisticamente significativa $(p>0,05)$ entre as forças desses últimos dois grupos de elásticos.

Os valores da força remanescente obtidos na leitura após 24 horas de ativação, para os elásticos curtos, médios e longos, foram, respectivamente, 193,25gf, 182,25gf e 185,75gf. Da mesma forma, a análise estatística evidenciou diferença significativa entre as magnitudes de força dos elásticos do grupo Curto quando comparada à dos grupos Médio e Longo.

Uma semana após o início da mensuração, os valores médios verificados para a força liberada pelos elásticos curtos, médios e longos foram, respectivamente, 148,75gf, 148,25gf e 149,50gf. A análise da variância não paramétrica (teste de Kruskal-Wallis), ao nível de significância de 0,05 , indicou evidência de homogeneidade $(p>0,05)$ entre as forças remanescentes dos três grupos de elásticos. Portanto, não houve diferenças estatisticamente significativas en- 
tre os grupos de elásticos quanto à força remanescente uma semana após o início do processo.

Após duas semanas, foram lidos valores médios de 136,25gf, 130,75gf e 134gf para os elásticos dos grupos Curto, Médio e Longo, respectivamente. Da mesma forma, o teste de Kruskal-Wallis, ao nível de significância de 0,05 , indicou evidência de homogeneidade $(p>0,05)$ entre as forças remanescentes dos três grupos de comprimento de elásticos, não havendo, portanto, diferenças estatisticamente significativas entre os grupos de elásticos quanto à força remanescente duas semanas após o início do processo.

No momento correspondente à terceira semana verificou-se, com significância estatística $(p<0,05)$, que a força liberada pelos elásticos curtos, 112,25gf, é menor do que a liberada pelos elásticos médios e longos, 116,25gf e 120gf, respectivamente. Porém, não foi encontrada diferença estatisticamente significativa $(p>0,05)$ entre as forças remanescentes liberadas por esses dois últimos grupos de elásticos.

Após a análise estatística dos valores correspondentes ao momento quatro semanas, conclui-se que as forças remanescentes liberadas pelos elásticos são todas diferentes umas das outras, sendo que no grupo Longo a força remanescente $(109,75 \mathrm{gf})$ é estatisticamente maior $(\mathrm{p}<0,05)$ do que no grupo Curto (102,75gf); a qual, por sua vez, é estatisticamente maior $(\mathrm{p}<0,05)$ que a do grupo Médio $(96,25 \mathrm{gf})$.

\section{DISCUSSÃO}

A extensa literatura sobre as propriedades dos elásticos em cadeia é difícil de ser avaliada e comparada, devido à natureza variável dos métodos de investigação e às diferenças entre as configurações das diversas cadeias já testadas, além da impossibilidade de se saber a composição exata desses materiais, muitas vezes mantida em sigilo pelos fabricantes.

A exemplo das influências de mudanças ou da composição do meio e dos efeitos de procedimentos de desinfecção nas cadeias de elásticos, as forças liberadas e sua degradação, assim como o pré-estiramento, são variáveis que têm sido analisadas ${ }^{20}$. No entanto, parece impossível controlar todos os fatores capazes de influenciar em uma situação clínica ${ }^{23}$.

De acordo com alguns estudos $2,13,14,15$, a degradação da força liberada pelos materiais elásticos sintéticos, quando avaliada em meio úmido, é significativamente maior do que quando em ambiente seco; por conseguinte, os segmentos de elásticos em cadeia foram mantidos imersos em saliva artificial. Optou-se por manter a temperatura de $37 \pm 1^{\circ} \mathrm{C}$, por se tratar da temperatura corpórea e porque sabe-se que a temperatura influencia de forma significativa na degradação da força liberada pelos elásticos ${ }^{14,25}$. O pré-estiramento das cadeias elastoméricas não parece induzir melhoras significativas às propriedades mecânicas dos elásticos; portanto, esse procedimento não foi realizado ${ }^{6,16}$.

Não objetivou-se com essa pesquisa comparar elásticos em cadeia de diferentes fabricantes, mas sim avaliar o comportamento das diferentes configurações de cadeia (curto, médio e longo) de um mesmo fabricante. Portanto, os elásticos sintéticos aqui utilizados foram escolhidos por apresentarem amplo emprego e aceitação por parte dos ortodontistas e para adicionar conhecimento sobre o comportamento mecânico dos mesmos.

Alguns autores avaliaram o comportamento dos elásticos durante um período de três semanas $^{4,8,27}$; enquanto outros, de quatro semanas ou mais $^{7,11,16,17,23}$. Selecionou-se, aqui, o período de quatro semanas, pois coincide com o tempo frequente de intervalo entre as consultas ortodônticas.

Buscou-se padronizar a intensidade da força inicial liberada pelos elásticos; entretanto, sua variação não pôde ser evitada, tendo inclusive sido observada dentro do mesmo grupo, provavelmente devido à já reconhecida aleatoriedade das conformações espaciais assumidas pelas macromoléculas de polímeros ${ }^{9}$. Essa variação também foi percebida por outros autores ${ }^{1,11}$. O valor inicial adotado, em torno de 200gf, está dentro da faixa considerada adequada para o movimento ortodôntico em direção distal de 
dentes caninos nos casos de extração de primeiros pré-molares ${ }^{21,22,26}$.

Nos momentos seguintes ao inicial, a intensidade da força variou consideravelmente dentro de cada grupo, nos diferentes momentos. Tanto os elásticos do grupo Curto quanto dos grupos Médio e Longo apresentaram, desde a primeira hora, valores estatisticamente significativos quando comparados com os respectivos valores da força inicialmente liberada. Os percentuais de força remanescente em relação à inicial, para cada grupo de cadeia elastomérica estudada, em cada tempo do experimento, são representados no Gráfico 1 .

A queda, com o tempo, na magnitude da força empregada para a movimentação distal de dentes caninos ocorre em função de dois fatores: o deslocamento relativo das unidades dentárias componentes da ancoragem; e a própria degradação das cadeias elastoméricas ${ }^{12}$. Portanto, parece plenamente justificada a diminuição do espaço entre os ganchos do dispositivo visando simular o efeito do movimento dentário sobre a diminuição da força dos elásticos.

A partir de estudos já realizados, sabe-se que, geralmente, os elásticos em cadeia perdem grande quantidade da força inicialmente liberada durante o primeiro dia decorrido de sua ativação, diminuição que pode ser de até $75 \%^{13,18}$. Consequentemente, Andreasen e Bishara ${ }^{1}$ sugerem a utilização de força

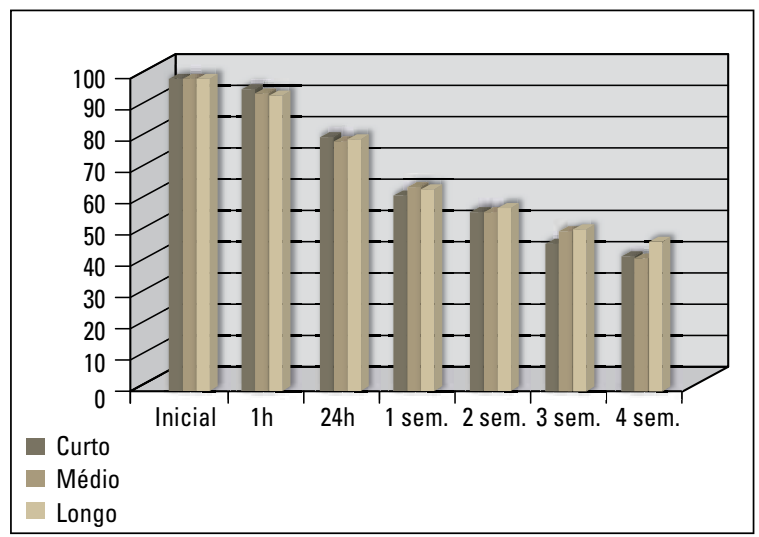

GRÁFICO 1 - Representação gráfica dos percentuais de força remanescente, para cada grupo das cadeias elastoméricas estudadas, em cada momento do experimento, em relação ao inicial. inicial 40\% maior do que a força considerada ótima para uma determinada movimentação ortodôntica, visando compensar a perda que comumente ocorre nas primeiras 24 horas. Contudo, outros autores sugerem não haver diferença no resultado final do movimento dentário esperado quando forças de maior intensidade são aplicadas ${ }^{5}$. Além disso, a força aumentada poderia causar mais desconforto ao pacien$t^{27}$ e outras complicações como reabsorção óssea solapante ${ }^{28}$. No presente estudo, aproximadamente $20 \%$ da força inicial dissiparam-se nesse mesmo período, evidenciando diferenças entre os produtos de diferentes fabricantes, bem como entre as variáveis estabelecidas em cada metodologia empregada.

Com relação à degradação da força em função do tempo, verificou-se que os três grupos de elásticos apresentaram considerável redução na intensidade da força liberada, chegando ao final do experimento com um percentual médio variando em torno de $55 \%$ de diminuição de força em relação à inicial (Gráf. 1). Deve-se notar que a partir da terceira semana as forças remanescentes parecem não mais estar dentro da faixa considerada ótima para o movimento dentário ortodôntico ${ }^{22,26}$. Entretanto, de acordo com os resultados observados por Boester e Johnston ${ }^{5}$, ainda é possível movimentar caninos em direção distal com essa força, mesmo que mais lentamente.

Embora os elásticos do grupo Curto tenham apresentado valor médio de força inicial maior que os demais, ao final do experimento o maior valor foi verificado no grupo Longo. Esses resultados diferem dos de outros estudos ${ }^{4,8}$ que concluíram que os elásticos curtos, sem espaçamento entre os elos, mantêm maior percentual de força ao longo do tempo.

Os valores percentuais médios verificados para a degradação da força nos diferentes intervalos de tempo pesquisados diferem dos de outros estudos, possivelmente em virtude dos diferentes fabricantes de elastômeros usarem composições variadas. Além disso, vale ressaltar as variações entre as metodologias empregadas, bem como limitações decorrentes dos detalhes das configurações já 
testadas, como a coloração e o espaçamento entre os elos das cadeias elastoméricas.

Importa acrescentar que, apesar de verificados alguns resultados estatisticamente significativos para a comparação da magnitude das forças liberadas em cada momento, entre os grupos Curto, Médio e Longo, essas diferenças não parecem ter expressão clínica, pois a maior diferença observada foi de aproximadamente $12 \mathrm{gf}$ (entre os grupos Curto e Médio), não considerada clinicamente suficiente a ponto de interferir no movimento dentário resultante.

\section{CONCLUSÕES}

Após a análise dos resultados das comparações entre os grupos de elásticos em cadeia Curto, Médio e Longo, foi possível concluir que o espaçamento entre os elos não parece representar uma característica clinicamente significativa na degradação da força ao longo do tempo. Os três grupos de elásticos apresentaram considerável redução na intensidade da força liberada, chegando ao final do experimento com um percentual médio em torno de $55 \%$ de diminuição em relação à força inicial. Aconselha-se que o intervalo entre as consultas de substituição dos segmentos de elásticos cinza em cadeia - de qualquer uma das três configurações da marca estudada - seja de três a quatro semanas, o que é sustentado pela literatura e pelos resultados aqui encontrados e testados para a simulação de movimentação dentária em casos de fechamento de espaço após exodontia de primeiro pré-molar e intenção de movimentação de canino em direção distal.

\title{
In vitro evaluation of force delivered by elastic chains
}

\begin{abstract}
Objective: To evaluate and compare, in vitro, the decrease in the forces delivered in three groups of elastic chains according to the distance between links (short, medium, long) and produced by the same manufacturer. Methods: The segments of elastic chains were stretched and kept activated during the trial using a device developed especially for this purpose, which also allowed force readings. Force degradation was evaluated by measuring force along time and calculating the percentage of force differences from initial force at each time interval and for each specimen under test. Results and Conclusions: Data were analyzed statistically and results showed that at the different time points after initial readings, force intensity varied within and between groups. Readings of remaining forces at each time moment compared with the initial reference force revealed statistically significant differences in all the comparisons in each group of elastics (short, medium, long). Although the comparisons between forces delivered at each time point revealed statistically significant differences, these differences do not seem to have a clinical significance. The space between links does not seem to be a clinically significant characteristic in force degradation along time.
\end{abstract}

Keywords: Orthodontic appliances. Dental materials. Elastomers. 


\section{REFERÊNCIAS}

1. Andreasen GF, Bishara S. Comparison of alastik chains of elastics involved with intra-arch molar-to-molar forces. Am J Orthod. 1971;60(2):200-1.

2. Ash JL, Nikolai RJ. Relaxation of orthodontic elastomeric chains and modules in vitro and in vivo. J Dent Res. 1978;57(5-6):685-90.

3. Baty DL, Storie DJ, von Fraunhofer JA. Synthetic elastomeric chains: a literature review. Am J Orthod Dentofacial Orthop. 1994;105(6):536-42.

4. Baty DL, Volz JE, von Fraunhofer JA. Force delivery properties of colored elastomeric modules. Am J Orthod Dentofacial Orthop. 1994;106(1):40-6.

5. Boester $\mathrm{CH}$, Johnston LE. A clinical investigation of the concepts of differential and optimal force in canine retraction. Angle Orthod. 1974;44(2):113-9.

6. Brantley WA, Salander S, Myers CL, Winders RV. Effects of prestretching on force degradation characteristics of plastic modules. Angle Orthod. 1979;49(1):37-43.

7. Chimenti C, Lecce D, Santucci L, Parziale V, Lucci M. In vitro assessment of elastomeric chain behavior. Prog Orthod. 2001;2(1):42-5.

8. De Genova DC, Mclnnes-Ledoux P, Weinberg R, Shaye R. Force degradation of orthodontic elastomeric chains: a product comparison study. Am J Orthod. 1985;87(5):377-84.

9. Eliades T, Eliades G, Silikas N, Watts DC. Tensile properties of orthodontic elastomeric chains. Eur J Orthod. 2004;26(2):157-62.

10. Ferreira Neto JJ, Caetano MTDO. A degradação da força de segmentos de elásticos em cadeia de diferentes tamanhos: estudo comparativo in vitro. J Bras Ortodon Ortop Facial. 2004;9(51):225-33.

11. Hershey HG, Reynolds WG. The plastic module as an orthodontic tooth-moving mechanism. Am J Orthod. 1975;67(5):554-62.

12. Howard RS, Nikolai RJ. On the relaxation of orthodontic elastic threads. Angle Orthod. 1979;49(3):167-72.

13. Huget EF, Patrick KS, Nunez LJ. Observations on the elastic behavior of a synthetic orthodontic elastomer. J Dent Res. 1990;69(2):496-501.

14. Hwang CJ, Cha JY. Mechanical and biological comparison of latex and silicone rubber bands. Am J Orthod Dentofacial Orthop. 2003;124(4):379-86.
15. Kanchana P, Godfrey K. Calibration of force extension and force degradation characteristics of orthodontic latex elastics. Am J Orthod Dentofacial Orthop. 2000;118(3):280-7.

16. Kim KH, Chung $\mathrm{CH}$, Choy $\mathrm{K}$, Lee JS, Vanarsdall RL. Effects of prestretching on force degradation of synthetic elastomeric chains. Am J Orthod Dentofacial Orthop. 2005;128(4):477-82.

17. Kuster R, Ingervall B, Bürgin W. Laboratory and intra-oral tests of the degradation of elastic chains. Eur J Orthod. $1986 ; 8(3): 202-8$

18. Lu TC, Wang WN, Tarng TH, Chen JW. Force decay of elastomeric chain-a serial study. Part II. Am J Orthod Dentofacial Orthop. 1993;104(4):373-7.

19. Matta ENR, Chevitarese O. Avaliação laboratorial da força liberada por elásticos plásticos. Rev Soc Bras Ortod. 1997;3(4):131-6.

20. Mayberry D, Allen R, Close J, Kinney DA. Effects of disinfection procedures on elastomeric ligatures. J Clin Orthod. 1996;30(1):49-51.

21. Quinn RS, Yoshikawa DK. A reassessment of force magnitude in orthodontics. Am J Orthod. 1985;88(3):252-60.

22. Reitan K. Some factors determining the evaluation of forces in orthodontics. Am J Orthod. 1957;43(1):32-45.

23. Rock WP, Wilson HJ, Fisher SE. Force reduction of orthodontic elastomeric chains after one month in the mouth. Br J Orthod. 1986;13(3):147-50.

24. Sonis AL, Van der Plas E, Gianelly A. A comparison of elastomeric auxiliaries versus elastic thread on premolar extraction site closure: an in vivo study. Am J Orthod. 1986;89(1):73-8

25. Stevenson JS, Kusy RP. Force application and decay characteristics of untreated and treated polyurethane elastomeric chains. Angle Orthod. 1994;64(6):455-64; discussion 465-7.

26. Storey E, Smith R. Force in orthodontics and its relation to tooth movement. Aust J Dent. 1952;56(2):11-8.

27. Wong AK. Orthodontic elastic materials. Angle Orthod. 1976;46(2):196-205.

28. Young J, Sandrik JL. The influence of preloading on stress relaxation of orthodontic elastic polymers. Angle Orthod. 1979;49(2):104-9. 